\title{
Torsion Dependence of Domain Transition and MI Effect of Melt-Extracted Co ${ }_{68.15} \mathrm{Fe}_{4.35} \mathrm{Si}_{12.25} \mathrm{~B}_{13.25} \mathrm{Nb}_{1} \mathrm{Cu}_{1}$ Microwires
}

\author{
Dawei Xing, ${ }^{1}$ Dongming Chen,, ${ }^{1}$ Fang Liu, ${ }^{1}$ Jingshun Liu, ${ }^{2}$ Hongxian Shen, ${ }^{1}$ Zhiliang Ning, \\ Fuyang Cao, ${ }^{1}$ and Jianfei Sun ${ }^{1}$ \\ ${ }^{1}$ School of Materials Science and Engineering, Harbin Institute of Technology, No. 92 West Dazhi Street, Harbin 150001, China \\ ${ }^{2}$ School of Materials Science and Engineering, Inner Mongolia University of Technology, No. 49 Aimin Street, Hohhot 010051, China
}

Correspondence should be addressed to Jianfei Sun; jfsun_hit@263.net

Received 16 October 2014; Revised 19 January 2015; Accepted 28 January 2015

Academic Editor: You Song

Copyright (C) 2015 Dawei Xing et al. This is an open access article distributed under the Creative Commons Attribution License, which permits unrestricted use, distribution, and reproduction in any medium, provided the original work is properly cited.

We present the torsional stress induced magnetoimpedance (MI) effect and surface domain structure evolution of magnetostrictive melt-extracted $\mathrm{Co}_{68.15} \mathrm{Fe}_{4.35} \mathrm{Si}_{12.25} \mathrm{~B}_{13.25} \mathrm{Nb}_{1} \mathrm{Cu}_{1}$ microwires. Experimental results indicate that the surface domain structures observed by magnetic force microscope (MFM) transform from the weak circumferential domain of as-cast state to the helical domain under large torsional strain of $81.6(2 \pi \mathrm{rad} / \mathrm{m})$. Domain wall movement distorts at torsional strain $\xi=20.4(2 \pi \mathrm{rad} / \mathrm{m})$ and forms a helical anisotropy with an angle of around $30^{\circ}$ versus axial direction of wire. At $15 \mathrm{MHz}$, the maximum of GMI ratio $\Delta Z / Z(\%)$ increases to $194.4 \%$ at $\xi=20.4(2 \pi \mathrm{rad} / \mathrm{m})$ from $116.3 \%$ of the as-cast state and then decreases to $134.9 \%$ at $\xi=102.0$ $(2 \pi \mathrm{rad} / \mathrm{m})$. The torsion magnetoimpedance (TMI) ratio $(\Delta Z / Z)_{\xi}(\%)$ is up to $290 \%$. Based on this large torsional strain and high $\mathrm{MI}$ ratio, the microwire can be as an referred candidate for high-performance TMI sensor application.

\section{Introduction}

The ferromagnetic microwires have been recognized as possessing much more promising magnetic sensing materials, especially for giant magnetoimpedance (GMI) effect, which has attracted considerable interest not only from the basically theoretical viewpoint but also from its wide technical applicability [1-4]. As well known, the near-zero magnetostrictive wires with soft magnetic properties have high MI ratios $[1,5]$. As one method of the amorphous microwire fabrication technologies, different from glass-coated spinning and in-rotating water quenching techniques, the melt-extraction technique consists of the following: (i) it has the largest solidification or cooling rate, which enables the wires to possess an excellent soft magnetic property; (ii) the melt-extracted wires without glass covering are more suitable for electronic package and GMI sensor applications; (iii) the parameters (i.e., linear velocity of wheel, feed rate of the molten, etc.) of meltextraction can be conveniently controlled to produce wires with uniform diameter and roundness $[6,7]$. In another perspective, near-zero magnetostrictive amorphous wires, with low resistivity $\rho$, high magnetic permeability $\mu_{\varphi}$, high saturation magnetization $M_{s}$, and small ferromagnetic resonance (FR) in the high-frequency range, possess outstanding GMI performance. Based on this principle, the element $\mathrm{B}$ in $\mathrm{CoFeSiB}$ alloy is substituted for $\mathrm{Nb}$ and $\mathrm{Cu}$ with low resistivity materials and a small amount of atomic percentage $1 \%$ and $1 \%$, respectively.

In the meantime, in the intermediate frequency range $(100 \mathrm{kHz} 20 \mathrm{MHz})$, GMI originates mainly from the variation of the skin depth owing to the strong changes of the effective magnetic permeability resulting in both domain wall motion and magnetization rotation; namely, it can be denoted by $\mu_{\varphi}=\mu_{\text {wall }}+\mu_{\text {rot }}[1,8]$. The role of $\mu_{\text {rot }}$ gives rise to the helical anisotropy change. In order to understand deeply the role of the helical anisotropy to the surface domain and GMI performance of the near-zero magnetostrictive meltextracted Co-based amorphous microwires, the imposed torsional strain on the microwires is necessary to observe the domain structure by magnetic force microscope (MFM) 
and modify the domain transition for MI and torsion magnetoimpedance (TMI) sensor application.

Up to now, much works have previously reported the influence of torsion on MI effect for Co-based and Febased glass-coated or in-rotating water amorphous wires [913]. Betancourt and Valenzuel found the $\mu_{\varphi}$ an asymmetric behavior dependent on the direction of the applied torsion strain of in-rotating water $\left(\mathrm{Co}_{94} \mathrm{Fe}_{6}\right)_{72.5} \mathrm{~B}_{15} \mathrm{Si}_{12.5}$ wires [9]. Garcia et al. studied the magnetoimpedance response under tensile and torsional stress of $\mathrm{Fe}_{76} \mathrm{~B}_{13} \mathrm{Si}_{11}$ glass-coated amorphous microwires and induced a helical magnetic anisotropy that modifies the magnetic domain structure and the magnetic response of the sample applied torsion stresses [10]. Popov et al. proposed the "enhanced core-shell" model of vanishing-magnetostrictive glass-coated Co-rich microwires to resolve the physical origin of the low-field hysteresis and the dependence of induced anisotropy field on the applied tensile and/or torsional stress [11]. However, only a few researchers resorted to the surface domain structure (SDM) of twisted microwire to explore the detailed information of the modified domain and the helical anisotropy.

As well known, the core-shell magnetic structures of nearly zero magnetostrictive melt-extracted Co-based amorphous wires consist of a cylindrical inner core domain oriented axially and the outer shell domain for circumferential magnetization, while the exchange energy tilts it towards a helical orientation [14]. Generally, GMI effect is very sensitive to the magnetic structure and the local anisotropies of soft magnetic materials. It It can be additionally served as a powerful tool to explore the important information of the magnetic structure and local anisotropies. Moreover, the domain structure and anisotropy field of the magnetic materials can be derived from analyzing the corresponding changes of GMI property of samples subjected to stress applied. The modification of GMI response is the consequence of the competition between the helical anisotropy introduced by the applied torsional stress and the internal anisotropies. Therefore, torsional stresses were found to modify the magnetization cycle of the wire [15], Young's modulus [16] and MI response [11], and so forth.

The aim of this work is conducted to study the static surface magnetic domain structure, MI effect of a nearly zero magnetostrictive melt-extracted Co-rich amorphous microwire dependence on applied extremely huge torsional strain and to obtain large MI value by modulating the magnetic structure and local anisotropies of soft magnetic microwires for potentially exploring the torsion MI sensors.

\section{Experimental}

We have prepared softly magnetic amorphous microwires of $\mathrm{Co}_{68.15} \mathrm{Fe}_{4.35} \mathrm{Si}_{12.25} \mathrm{~B}_{13.25} \mathrm{Nb}_{1} \mathrm{Cu}_{1}$ with diameter of $35 \mu \mathrm{m}$ by a modified melt-extraction technique [6]. Impedance measurement is carried out at the intermediate frequency range of $100 \mathrm{kHz} 15 \mathrm{MHz}$ by using Agilent 4294A precision impedance analyzer. The MR, MX, and MI ratios are defined as follows, respectively:

$$
\frac{\Delta R}{R}=\frac{\left\langle\left(R(H)-R\left(H_{\max }\right)\right)\right\rangle}{R\left(H_{\max }\right)} \times 100 \%,
$$

$$
\begin{aligned}
& \frac{\Delta X}{X}=\frac{\left\langle\left(X(H)-X\left(H_{\max }\right)\right)\right\rangle}{X\left(H_{\max }\right)} \times 100 \%, \\
& \frac{\Delta Z}{Z}=\frac{\left\langle\left(Z(H)-Z\left(H_{\max }\right)\right)\right\rangle}{Z\left(H_{\max }\right)} \times 100 \% .
\end{aligned}
$$

The total length of each microwire is $58 \mathrm{~mm}$ under the application of a torsional strain $\xi$ ranging from 0 to 102.0 $(2 \pi \mathrm{rad} / \mathrm{m})$ with the one end fixed and the other end rotating in clockwise direction. Then, we selected the central part of $18 \mathrm{~mm}$ long microwire to connect to the circuit and to measure the MI effect by the driving amplitude of $20 \mathrm{~mA}$ with DC axial field applied by using a pair of Helmholtz coils. The external field is up to the maximum of $80 \mathrm{Oe}$. The Helmholtz coil was kept perpendicular to the earth's magnetic field direction.

The torsion magnetoimpedance (TMI) ratio, $(\Delta Z / Z)_{\xi}$, or the relative change of the maximum magnetoimpedance with the torsion is expressed as follows:

$$
\left(\frac{\Delta Z}{Z}\right)_{\xi}=\frac{\left\langle\left(Z_{\max }(\xi)-Z_{\max }\left(\xi_{\max }\right)\right)\right\rangle}{Z_{\max }\left(\xi_{\max }\right)} \times 100 \%,
$$

where $\xi_{\max }=102.0(2 \pi \mathrm{rad} / \mathrm{m})$ and the external field $H$ is 0 Oe.

Domain observation of microwire is conducted by a Nanoscope III multimode atomic force microscope from Digital Instruments. The micro-etched silicon probe tip is to collect related information by using a combination of tapping and lift mode. During lift mode, the magnetic data were collected and displayed.

\section{Results and Discussion}

It has been known that the changes of both resistance $R$ and reactance $X$ depending on $\mu_{\varphi}$ and external field $H$ will alter the impedance $Z$ and therefore are contributed to GMI behavior [1]. Based on the expression of the impedance $Z$ of cylindrical magnetic conductor, $Z=R_{\mathrm{dc}} k a J_{0}(k a) / 2 J_{1}(k a)$ with $k=(1+i) / \delta_{m}$ is expressed in terms of first order Bessel functions $J_{0}, J_{1}$ and the material parameters: the skin depth $\delta_{m}$, the radius $a$, and the dc resistance $R_{\mathrm{dc}}$ of the conductor. The skin depth $\delta_{m}, \delta_{m}=\left(\rho / \pi \mu_{\varphi} f\right)^{1 / 2}$, where $\rho$ is the resistivity of the conductor and $f$ is the current frequency, and the $\mu_{\varphi}$ is circumferential permeability. The resistance $R$ and reactance $X$ can be depicted as: $R=\rho l / 2 \pi(a-$ $\left.\delta_{m}\right) \delta_{m} ; X=0.175 \mu_{0} l f\left\langle\mu_{\varphi}\right\rangle / \omega$, where $\mu_{0}$ and $\mu_{\varphi}$ are the vacuum permeability and the average relative circumferential permeability, respectively [1].

Figure 1 shows the zero-field frequency dependence of resistance $R(\mathrm{a})$, reactance $X(\mathrm{~b})$, and impedance $Z$ (c) of microwires with torsion strain from as-cast to 102.0 $(2 \pi \mathrm{rad} / \mathrm{m})$. An obvious monotonic increase in the resistance $R$ (as shown in Figure 1(a)), inductive reactance $X$ (Figure 1(b)), and impedance $Z$ (Figure 1(c)) is observed with the frequency range of $100 \mathrm{kHz} \sim 15 \mathrm{MHz}$ for the skin effect. And the maximum of each of $R, X$, and $Z$ values is $477.7 \Omega, 297.1 \Omega$, and $562.5 \Omega$, respectively, as the torsional strain increased to $20.4(2 \pi \mathrm{rad} / \mathrm{m})$. Continued to increase 


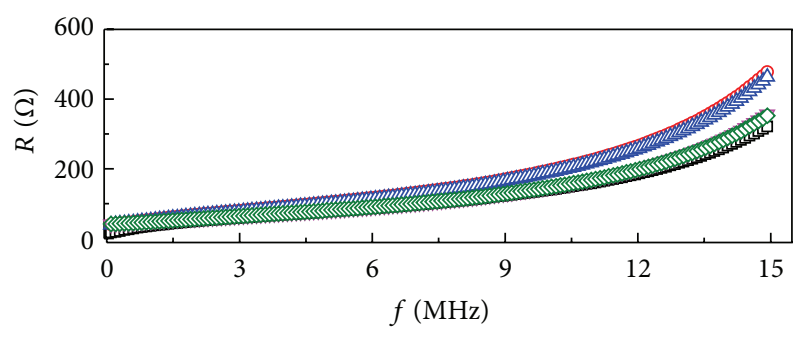

(a)

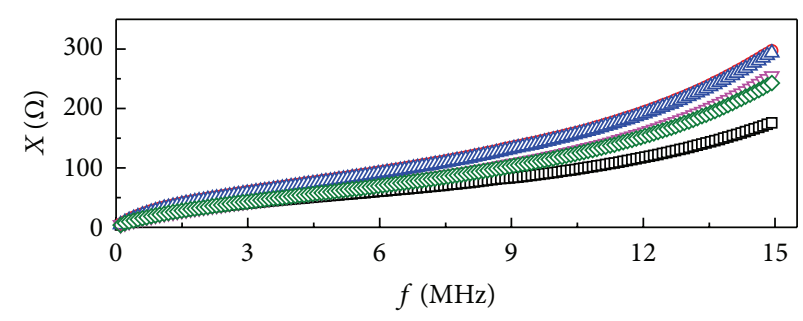

(b)

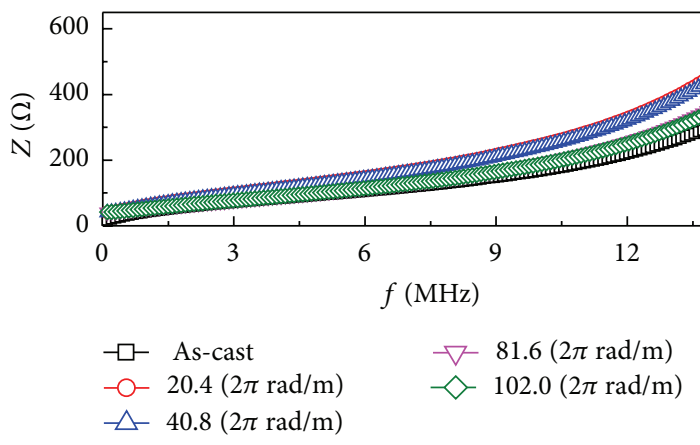

(c)

FIGURE 1: Frequency dependence of (a) resistance, (b) inductive reactance, and (c) impedance of microwires with different torsion stresses.

the torsion strain up to $40.8 \sim 102.0(2 \pi \mathrm{rad} / \mathrm{m})$, all the values of $R, X$, and $Z$ curves decrease. Additionally, the curves of $(R, X$, and $Z)$ at torsional strain of $20.4(2 \pi \mathrm{rad} / \mathrm{m})$ have a very small difference with curves of $40.8(2 \pi \mathrm{rad} / \mathrm{m})$. Also a similar behavior displays between curves of $81.2(2 \pi \mathrm{rad} / \mathrm{m})$ torsional strain with $102.0(2 \pi \mathrm{rad} / \mathrm{m})$ torsional strain. At the intermediate frequency regime, skin effect is dominant and the impedance is proportional to the square root of frequency and circumferential permeability; namely, $Z \propto\left(\omega \mu_{\varphi}\right)^{1 / 2}$. The influence of torsion stress on the microwire will improve the circular permeability $\mu_{\varphi}$ at a certain extent. Therefore, the corresponding values of $R, X$, and $Z$ for the torsional microwires all increase with the frequency.

Figure 2 reveals the magnetic field dependence of the magnetoresistance $\Delta R / R \%$ (Figure 2(a)), magnetoinductive reactance $\Delta X / X \%$ (Figure $2(\mathrm{~b})$ ), and magnetoimpedance $\Delta Z / Z \%$ (Figure 2(c)) ratios at $f=15 \mathrm{MHz}$ for applied torsion $\xi$ from 0 to $102.0(2 \pi \mathrm{rad} / \mathrm{m})$. In comparison with the behavior of $\Delta R / R \%$ and $\Delta Z / Z \%$, the curves of $\Delta X / X \%$ (Figure $2(\mathrm{~b})$ ) appear to the peaks with positions from $0.4 \mathrm{Oe}$ to $0.7 \mathrm{Oe}$ for torsion strain from $20.4(2 \pi \mathrm{rad} / \mathrm{m})$ to $102.0(2 \pi \mathrm{rad} / \mathrm{m})$. This torsion stress can be considered to change greatly the permeability. In addition, this response of the microwire is attributed to two magnetization processes: spin rotation and bulging of pinned domain walls. As shown in Figure 2(c), the curves of $\Delta Z / Z \%$ also exhibit the increasing trend at $\xi=20.4$ $(2 \pi \mathrm{rad} / \mathrm{m})$ and then decrease with the $\xi$ to $102.0(2 \pi \mathrm{rad} / \mathrm{m})$. The magnified plot of the peak value of $\Delta Z / Z \%$ inserted in the Figure 2(c) displays the ratio of $(\Delta Z / Z)_{\max } \%$ from $116.2 \%$ at as-cast to $194.4 \%$ at $\xi=20.4(2 \pi \mathrm{rad} / \mathrm{m})$ for $f=15 \mathrm{MHz}$. This enhanced improvement can be attributed to the releasing residual stress and the modified surface domain structure, as shown in Figure 3(b).

The behavior of GMI profiles is well resolved by considering the domain model with periodically circular domains [17]. Large $\mu_{\varphi}$ is contributed to the high GMI ratio and welldefined circumferential domain structure [18]. Therefore, we can improve the circumferential permeability $\mu_{\varphi}$ and impedance $Z$ by modulating the surface domain structure.

Accordingly, the surface morphology, domain structure obtained by MFM, and the schematic plot of microwire are presented for untwisted and under torsional strain state in Figure 3. The surface domain structure of the microwire of as-cast state is showed in Figure 3(a). The ill-defined circumferential domain configuration is exhibited with the average domain width of about $1.0 \mu \mathrm{m}$. The weak and uneven circumferential domain on the whole surface result from the microwire preparation process and the weak circular magnetic anisotropy. The residual stress and even heterogeneous microstructure of microwire all are not conducive to the formation of circumferential domain. So the corresponding the ratio of $(\Delta Z / Z \%)_{\max }$ is only $116.2 \%$. In order to induce helical anisotropy and further to influence the magneto-impedance response, each microwire was submitted to a homogenous torsional stress to form even domain structure. The modification of domain structure with increasingly applied torsion is obtained in Figures $3(\mathrm{~b}), 3(\mathrm{c})$, and $3(\mathrm{~d})$. For $\xi=20.4(2 \pi \mathrm{rad} / \mathrm{m})$, the surface morphology of the microwire appears distortion for the surface roughness of dozens of nanometers. Moreover, the domain structure was observed clearly with torsion stress applied to the wire in middle part of Figure 3(b). 


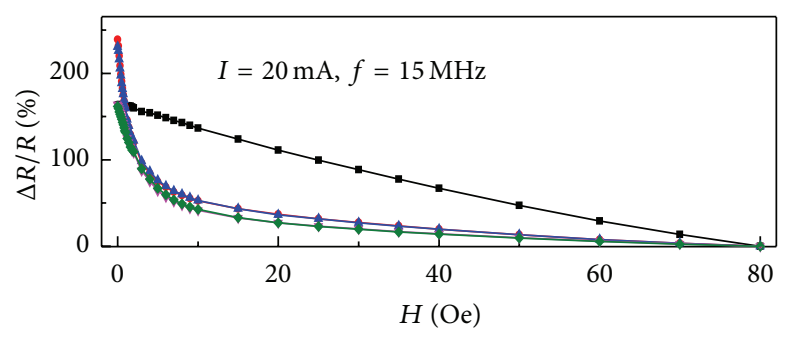

(a)

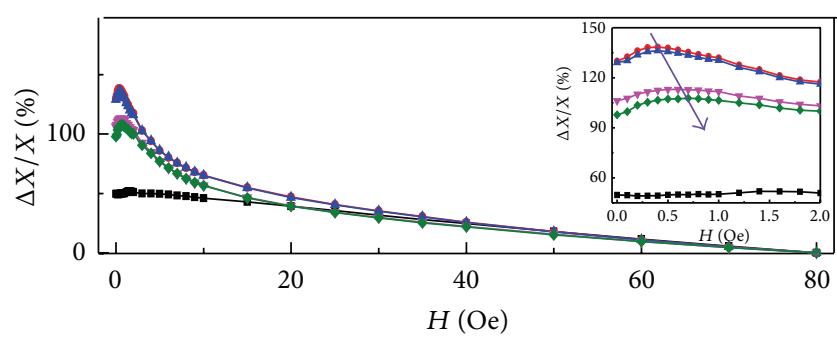

(b)

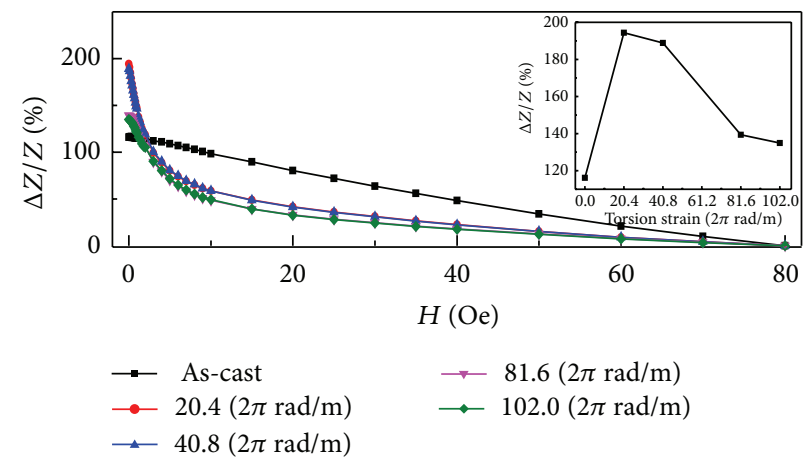

(c)

FiguRE 2: Magnetic field dependence of the magnetoresistance $(\Delta R / R)$, magnetoreactance $(\Delta X / X)$, and magnetoimpedance $(\Delta Z / Z)$ ratios at $f=15 \mathrm{MHz}$ for selectively applied torsion. And insets of the magnified peak positions of $\Delta X / X$ in (b) and of the maximum values of $\Delta Z / Z$ dependence to torsion strain in (c).

Different from the weak circumferential domain of as-cast state, the surface domain with the torsional state $\xi=20.4(2 \pi \mathrm{rad} / \mathrm{m})$ turns to form the zigzag domain, which is derived from the helical anisotropy. The relatively large GMI ratio on this stage is contributed from the enhanced circumferential anisotropy generated by circular component of torsion stress. Additionally, the circumferential component of helical anisotropy induced by applied the torsional stress improves the circular anisotropy, which is benefit for circumferential permeability $\mu_{\varphi}$, further for GMI ratios improvement. Consequently, the average domain width increases to $1.1 \mu \mathrm{m}$ at $\xi=20.4(2 \pi \mathrm{rad} / \mathrm{m})$. For this circumstance, the circumferential domain turns to zigzag domain under the torsional strain, at which behavior is different from the consistency deflection for positive magnetostrictive Fe-based wires [19]. As the applied torsion increases to $61.2(2 \pi \mathrm{rad} / \mathrm{m})$ in Figure $3(\mathrm{c})$, the uneven domain is changed into more clear domain and smooth domain wall with average width about $0.9 \mu \mathrm{m}$ and tilt angle $\theta$ up to about $67^{\circ}$. Further to apply the torsional strain to $81.6(2 \pi \mathrm{rad} / \mathrm{m})$, the uniform helical domain is obtained with domain wall angle $\theta \approx 30^{\circ}$. Based on the extreme torsion applied, GMI profile exhibits the maximum of $139.4 \%$ at $\xi=81.6(2 \pi \mathrm{rad} / \mathrm{m})$. The slight difference between GMI ratios of $\xi=81.6(2 \pi \mathrm{rad} / \mathrm{m})$ and $\xi=102.0$ $(2 \pi \mathrm{rad} / \mathrm{m})$ indicates the surface domain in each case is very similar. Momently, a uniform helical domain is therefore formed with smaller domain wall angle of $\theta \approx 30^{\circ}$, at which performance manifests the homogeneous and larger helical anisotropy. Additionally, an interesting result of
Co-based melt-extracted microwires with large torsion strain performance of $\xi=102.0(2 \pi \mathrm{rad} / \mathrm{m})$ will be as the ideal candidate potential materials for high-performance torsion TMI sensor [20].

To sum up, the torsional strain $\xi$ dependence of torsional impedance profile $(\Delta Z / Z)_{\xi}$ and domain wall angle $\theta$ is illustrated in Figure 4. As observed, the $(\Delta Z / Z)_{\xi}$ ratios decrease with the increasing torsional strain $\xi$. The maximum ratio $(\Delta Z / Z)_{\xi}$ is up to $290 \%$. Combining with the domain transformation applied different torsional strain (Figure 3), the domain wall movement is neither ideally towards longitudinal nor uniformly with a helical component along the axial direction. The domain wall distortion firstly is perpendicular to the axial direction (Figure 3(b)) and then tilts to transverse direction with a small angle (Figure 3(c)) and forms a helical magnetic anisotropy making an angle about $30^{\circ}$ with the longitudinal direction (Figure 3(d)). So the large torsional strain is up to $102.0(2 \pi \mathrm{rad} / \mathrm{m})$ as the candidate for torsion magnetoimpedance (TSI) application.

\section{Summary}

In conclusion, we have systematically investigated the surface domain transition and MI effect of Co-based melt-extracted microwires at different torsional strain. Firstly, the Co-based microwire is revealed to achieve the huge torsion strain behavior of $\xi=102.0(2 \pi \mathrm{rad} / \mathrm{m})$. We also observed an increased MI ratio as a result of modification of the helical magnetic anisotropy. Accordingly, the domain transformation under torsion stress corresponding to the GMI profile 

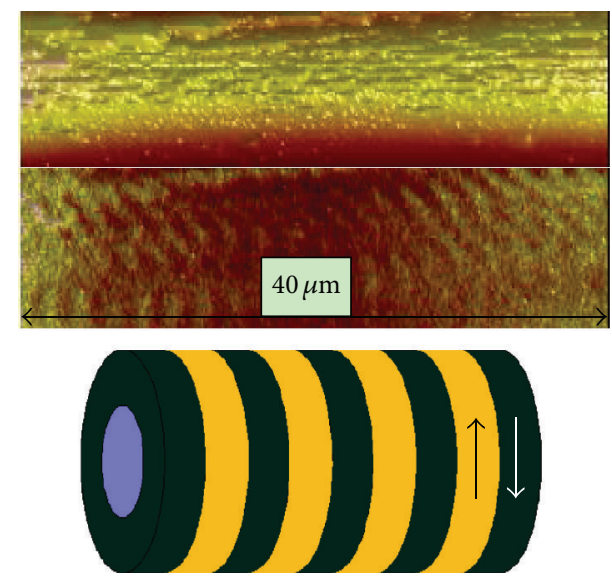

(a)
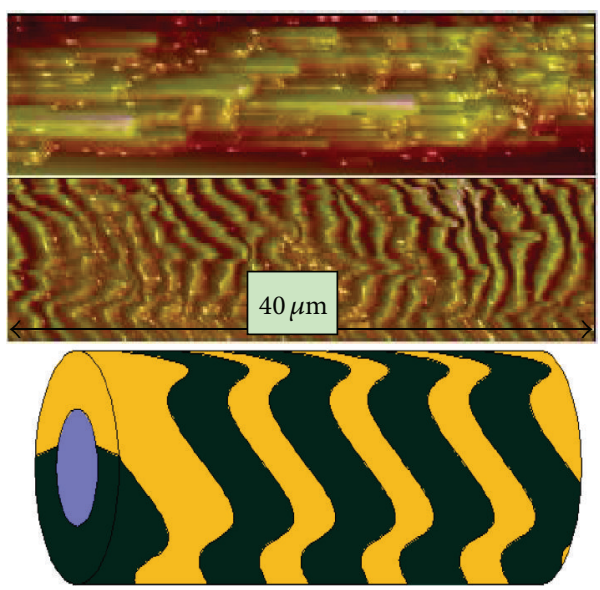

(c)
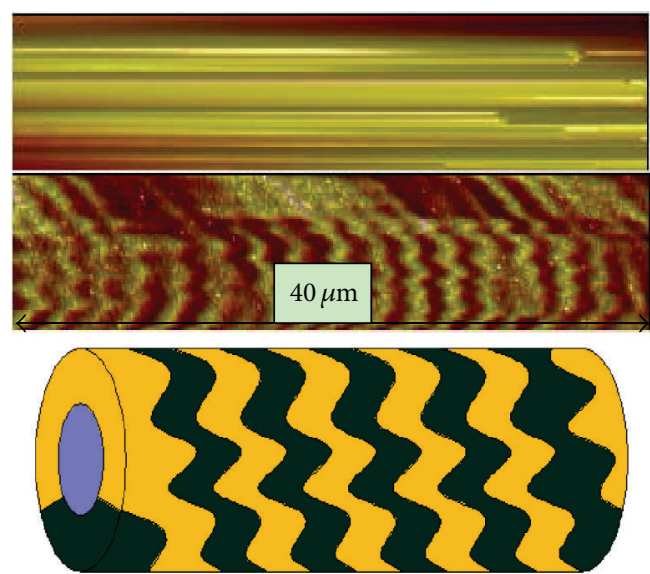

(b)
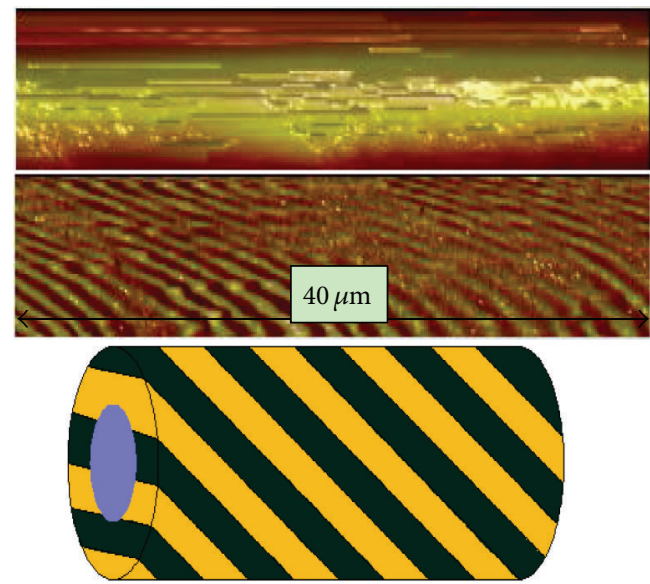

(d)

FIGURE 3: Surface domain structures transition and corresponding schematic diagrams of $\mathrm{Co}_{68.15} \mathrm{Fe}_{4.35} \mathrm{Si}_{12.25} \mathrm{~B}_{13.25} \mathrm{Nb}_{1} \mathrm{Cu}_{1}$ amorphous wires: (a) as-prepared and under different values of the applied torsion in the clockwise sense: (b) $\xi=20.4(2 \pi \mathrm{rad} / \mathrm{m}),(\mathrm{c}) \xi=61.2(2 \pi \mathrm{rad} / \mathrm{m})$, and (d) $\xi=81.6(2 \pi \mathrm{rad} / \mathrm{m})$.

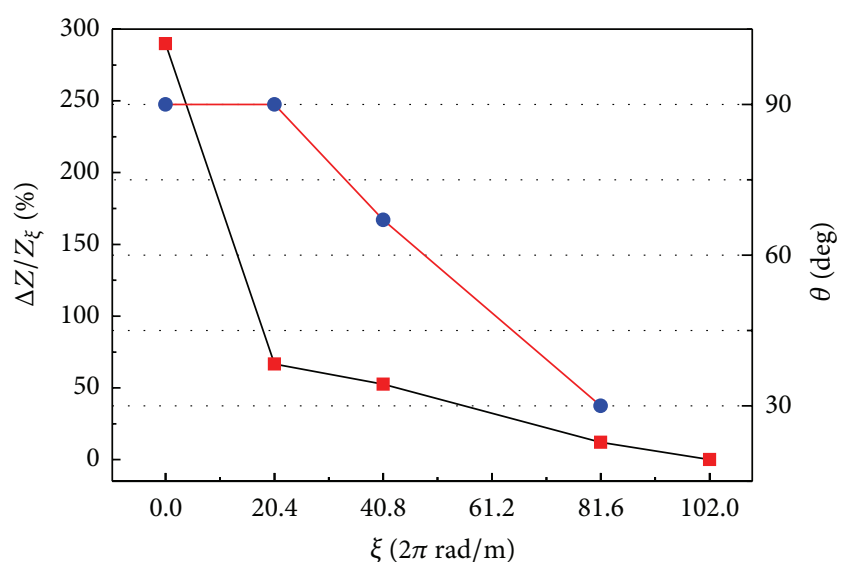

FIGURE 4: Torsional strain $\xi$ dependence of the maximum MI ratios and domain wall angle $\theta$.

exhibits the heterogeneous transition. And the obtained results on large torsion magnetoimpedance ratio $(\Delta Z / Z)_{\xi}$ to
$290 \%$ can be suitable for further development of the highperformance torque TSI sensors.

\section{Conflict of Interests}

The authors declare that there is no conflict of interests regarding the publication of this paper.

\section{Acknowledgments}

Dawei Xing acknowledges the financial support provided by the Natural Science Foundation of China (NSFC) under Grant no. 51371067. Jingshun Liu acknowledges the supports from NSFC with Grant no. 51401111, Natural Science Foundation of Inner Mongolia Autonomous Region of China under Grant no. 2014BS0503, and Scientific Research Foundation of the Higher Education Institutions (SRFHEI) of Inner Mongolia Autonomous Region of China under Grant no. NJZY14062. 


\section{References}

[1] M. H. Phan and H. X. Peng, "Giant magnetoimpedance materials: fundamentals and applications," Progress in Materials Science, vol. 53, no. 2, pp. 323-420, 2008.

[2] L. V. Panina and K. Mohri, "Magneto-impedance effect in amorphous wires," Applied Physics Letters, vol. 65, no. 9, pp. 1189-1191, 1994.

[3] F. X. Qin and H. X. Peng, "Ferromagnetic microwires enabled multifunctional composite materials," Progress in Materials Science, vol. 58, no. 2, pp. 183-259, 2013.

[4] A. Zhukov and V. Zhukova, Magnetic Properties and Applications of Ferromagnetic Mircowires with Amorphous and Nanocrystalline Structure, Nova Science Publishers, New York, NY, USA, 2009.

[5] M. Knobel and K. R. Pirota, "Giant magnetoimpedance: concepts and recent progress," Journal of Magnetism and Magnetic Materials, vol. 242-245, pp. 33-40, 2002.

[6] H. Wang, D. W. Xing, X. D. Wang, and J. F. Sun, "Fabrication and characterization of melt-extracted co-based amorphous wires," Metallurgical and Materials Transactions A, vol. 42, no. 4, pp. 1103-1108, 2011.

[7] J. Liu, F. Qin, D. Chen et al., "Combined current-modulation annealing induced enhancement of giant magnetoimpedance effect of co-rich amorphous microwires," Journal of Applied Physics, vol. 115, no. 17, Article ID 17A326, 2014.

[8] L. V. Panina, K. Mohri, T. Uchiyama, M. Noda, and K. Bushida, "Giant magneto-impedance in Co-rich amorphous wires and films," IEEE Transactions on Magnetics, vol. 31, no. 2, pp. 12491260, 1995.

[9] I. Betancourt and R. Valenzuel, "Effect of helical-induced anisotropy on the magnetoinductance response of Co-based amorphous wires," Applied Physics Letters, vol. 83, p. 2022, 2003.

[10] C. Garcia, A. Chizhik, A. Zhukov et al., "Influence of torsion and tensile stress on magnetoimpedance effect in Fe-rich amorphous microwires at high frequencies," Journal of Magnetism and Magnetic Materials, vol. 316, no. 2, pp. e896-e899, 2007.

[11] V. V. Popov, V. N. Berzhansky, H. V. Gomonay, and F. $\mathrm{X}$. Qin, "Stress-induced magnetic hysteresis in amorphous microwires probed by microwave giant magnetoimpedance measurements," Journal of Applied Physics, vol. 113, no. 17, Article ID 17A326, 2013.

[12] Y. F. Li, M. Vázquez, and D. X. Chen, "Torsion-dependent magnetoimpedance in $\mathrm{FeCuNbSiB}$ nanocrystalline wires with vanishing or transverse anisotropy," Journal of Applied Physics, vol. 93, no. 12, pp. 9839-9844, 2003.

[13] J. M. Blanco, A. Zhukov, A. P. Chen, A. F. Cobeno, A. Chizhik, and J. Gonzalez, "Asymmetric torsion giant impedance in nearly-zero magnetostrictive amorphous wires with induced helical anisotropy," Journal of Physics D: Applied Physics, vol. 34, no. 6, pp. L31-L34, 2001.

[14] P. Ciureanu, I. Khalil, L. G. C. Melo, P. Rudkowski, and A. Yelon, "Stress-induced asymmetric magneto-impedance in melt-extracted Co-rich amorphous wires," Journal of Magnetism and Magnetic Materials, vol. 249, no. 1-2, pp. 305-309, 2002.

[15] L. P. Shen, T. Uchiyama, K. Mohri, E. Kita, and K. Bushida, "Sensitive stress-impedance micro sensor using amorphous magnetostrictive wire," IEEE Transactions on Magnetics, vol. 33, no. 5, pp. 3355-3357, 1997.
[16] S. Atalay, P. T. Squire, and P. Rudkowski, "Magnetic and magnetoelastic properties of $\mathrm{Fe}-\mathrm{Si}-\mathrm{B}$ metallic fibers," IEEE Transactions on Magnetics, vol. 32, no. 5, pp. 4875-4877, 1996.

[17] D. X. Chen, J. Muñoz, A. Hernando, and M. Vázquez, "Magnetoimpedance of metallic ferromagnetic wires," Physical Review $B$, vol. 57, no. 17, pp. 10699-10704, 1998.

[18] D.-M. Chen, D.-W. Xing, F.-X. Qin et al., "Correlation of magnetic domains, microstructure and GMI effect of Joule-annealed melt-extracted $\mathrm{Co}_{68.15} \mathrm{Fe}_{4.35} \mathrm{Si}_{12.25} \mathrm{~B}_{13.75} \mathrm{Nb}_{1} \mathrm{Cu}_{0.5}$ microwires for double functional sensors," Physica Status Solid A, vol. 210, no. 11, pp. 2515-2520, 2013.

[19] B. Hernando, M. L. Sánchez, V. M. Prida et al., "Magnetic domain structure of amorphous $\mathrm{Fe}_{73.5} \mathrm{Si}_{13.5} B_{9} \mathrm{Nb}_{3} \mathrm{Cu}_{1}$ wires under torsional stress," Journal of Applied Physics, vol. 103, no. 7, Article ID 07E716, 2008.

[20] M. Tejedory, B. Hernandoy, M. L. Sánchez, V. M. Priday, and M. Vázquez, "The torsional dependence of the magnetoimpedance effect in current-annealed Co-rich amorphous wires," Journal of Physics D: Applied Physics, vol. 31, no. 23, pp. 3331-3336, 1998. 

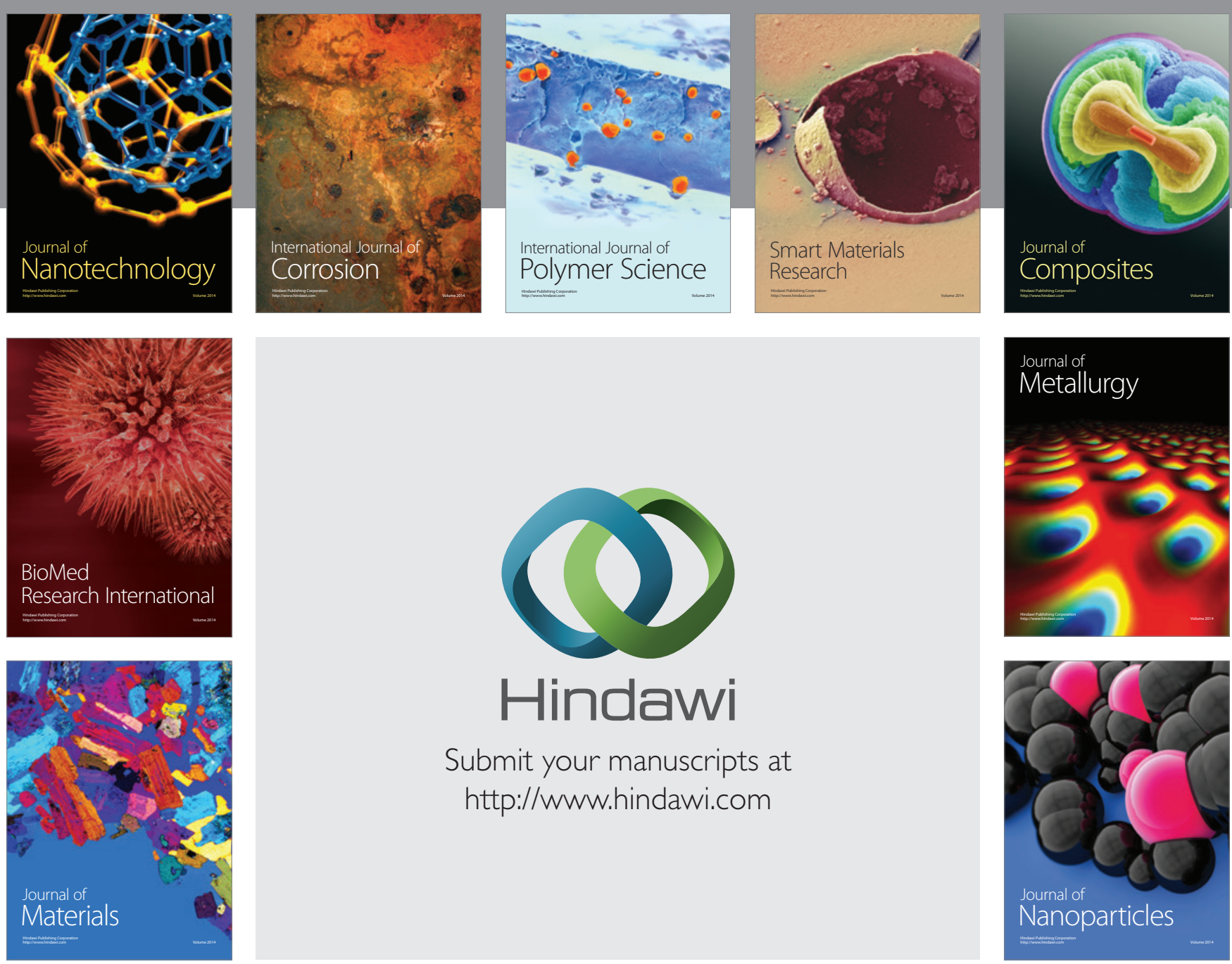

Submit your manuscripts at http://www.hindawi.com
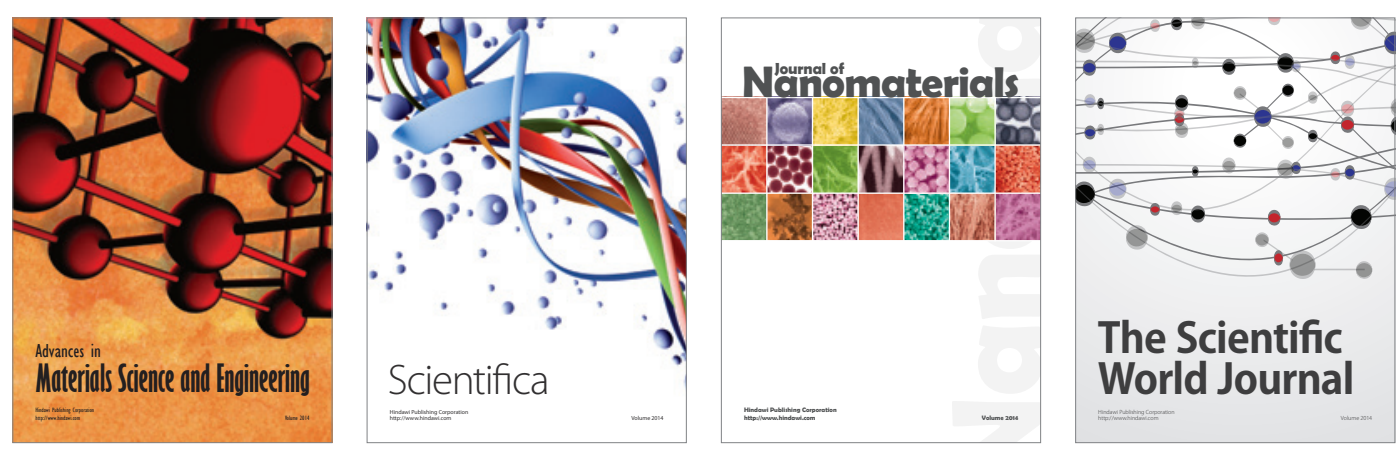

\section{The Scientific World Journal}
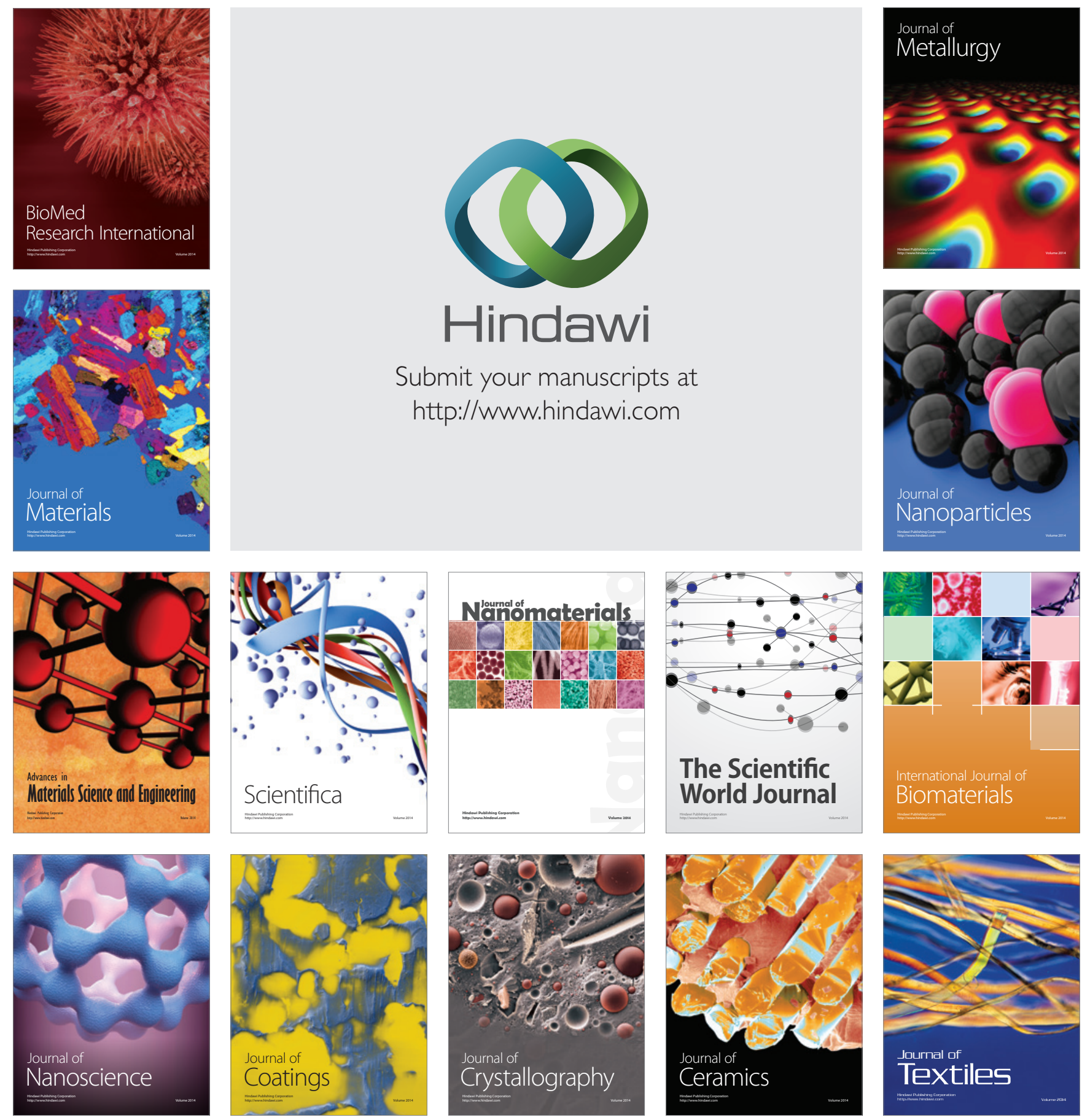